\title{
A Note on Four-Dimensional Symmetry Algebras and Fourth-Order Ordinary Differential Equations
}

\author{
A. Fatima, ${ }^{1}$ Muhammad Ayub, ${ }^{2}$ and F. M. Mahomed ${ }^{1}$ \\ ${ }^{1}$ Centre for Differential Equations, Continuum Mechanics and Applications, School of Computational and Applied Mathematics, \\ University of the Witwatersrand, Johannesburg 2050, South Africa \\ ${ }^{2}$ Department of Mathematics, COMSATS Institute of Information Technology, Abbottabad 22060, Pakistan
}

Correspondence should be addressed to A. Fatima; emanfatima81@yahoo.com and F. M. Mahomed; fazal.mahomed@wits.ac.za

Received 12 November 2012; Accepted 24 December 2012

Academic Editor: Mehmet Pakdemirli

Copyright (C) 2013 A. Fatima et al. This is an open access article distributed under the Creative Commons Attribution License, which permits unrestricted use, distribution, and reproduction in any medium, provided the original work is properly cited.

We provide a supplementation of the results on the canonical forms for scalar fourth-order ordinary differential equations (ODEs) which admit four-dimensional Lie algebras obtained recently. Together with these new canonical forms, a complete list of scalar fourth-order ODEs that admit four-dimensional Lie algebras is available.

\section{Introduction}

The integrability of scalar ordinary differential equations (ODEs) by use of the Lie symmetry method depends on their symmetrical Lie algebra if the Lie algebra is solvable and of sufficient dimension. There exists two different approaches to the integrability of differential equations using Lie point symmetries. One is the direct method in which Lie point symmetries are utilized to perform integrability by successive reduction of order of the equation using ideals of the algebra. The other approach is the canonical form method if the equations are classified into different types according to the canonical forms of the corresponding Lie algebra.

Lie [1] classified scalar second-order ODEs into four types on the basis of their admitted two-dimensional Lie algebras and also performed integration of the representative equations corresponding to the canonical forms of the twodimensional symmetry algebra. Therefore, scalar secondorder ODEs can be integrated by using canonical variables which map the symmetry generators to Lie's canonical forms. Also here one can use successive reduction of order by using ideals of the symmetry algebra (see, e.g., Olver [2]). The Noether equivalence approach for Lagrangians corresponding to scalar second-order ODEs is discussed in Kara et al. [3].
The canonical forms for scalar third-order ODEs that admit three symmetries were obtained by Mahomed and Leach [4]. Then Ibragimov and Nucci [5] provided the integrability of these canonical forms.

In their paper, Cerquetelli et al. [6] constructed realizations in the plane of four-dimensional Lie algebras listed by Patera and Winternitz [7]. Moreover, the classification of subalgebras of all real Lie algebras of dimension $\leq 4$ was discussed in [7]. The construction of Cerquetelli et al. [6] was based on the three-dimensional subalgebras provided in [7]. They invoked the realizations of three-dimensional Lie algebras in the plane derived earlier by Mahomed and Leach [8] for this purpose. They then determined the fourth-order ODEs admitting the realizations of the obtained four-dimensional algebras as their Lie symmetry algebra. Finally, they provided the route to the integration of the classified fourth-order ODEs. However, the derived realizations of four-dimensional Lie algebras need supplementation in the light of recent work by Popovych et al. [9]. Recently, these authors constructed a complete set of inequivalent realizations of real Lie algebras of dimension not greater than four in vector fields in the space of an arbitrary (finite) numbers of variables. We use the results of [9] to complete the classification of fourth-order ODEs in terms of their four-dimensional algebras presented in [6].

Apart from scalar fourth-order ODEs arising in the symmetry reductions of partial differential equations such as 
the linear wave equation in an inhomogeneous medium (see [10]), they occur prominently as model equations in the form of the static Euler-Bernoulli beam (see, e.g., [11]) and EmdenFowler equations. Such equations have been investigated for symmetry properties in $[12,13]$.

Firstly, we provide a comparison of the results of [9] and that of [6] related to the realizations of four-dimensional Lie algebras as vector fields in the plane. Then we list the new canonical forms of scalar fourth-order ODEs which possess four-dimensional algebras.

\section{Comparison of the Results of $[6,9]$}

We show here that the results on realizations of fourdimensional algebras in the plane given in [6] are a special case of the corresponding set of realizations given in [9]. We make a comparison of the lists of realizations given in [6] and [9]. It should be remarked that in general a result of classification of realizations may contain errors of two types, namely,

(i) missing of some inequivalent cases and

(ii) mutually equivalent cases.

In the following comparison, some first type of errors exist in [6]. Five cases are missing. There are some other cases which can be combined in a compact form and also some arbitrary parameters and functions need modification according to the results of [9] related to realizations in the plane. Below we keep the notations of both: on the left hand side the notations of [6] and on right hand side that of [9]. However, for the final results and further utilization, we keep the notations of [9].

2.1. Four-Dimensional Algebras. We use the nomenclature of Patera and Winternitz [7] in the naming of the algebras such as $4 A_{1}$. Thus we do not provide a table of the abstract algebras of dimension four as this is easily available.

Here the $N u$ refers to the realizations given in the work [6] and $R$ to that of [9].

(i) $N u\left(4 A_{1}\right) \sim\left(4 A_{1}\right)\left(\bar{e}_{1}=e_{2}, \bar{e}_{2}=e_{3}, \bar{e}_{3}=e_{1}, \bar{e}_{4}=e_{4}\right)$. $N u\left(4 A_{1}\right) \sim R\left(4 A_{1}, 11\right)(\bar{t}=y, \bar{x}=x)$.

(ii) $N u\left(A_{2} \oplus 2 A_{1}\right) \sim\left(A_{2.1} \oplus 2 A_{1}\right)\left(\bar{e}_{1}=e_{2}, \bar{e}_{2}=-e_{1}, \bar{e}_{3}=\right.$ $\left.e_{3}, \bar{e}_{4}=e_{4}\right)$. No realization exists in $(1+1)$-dimension.

(iii) $N u\left(2 A_{2}\right) \sim\left(2 A_{2.1}\right)\left(\bar{e}_{1}=e_{2}, \bar{e}_{2}=-e_{1}, \bar{e}_{3}=e_{4}\right.$, $\left.\bar{e}_{4}=-e_{3}\right)$.

$N u\left(2 A_{2}\right) \sim R\left(2 A_{2.1}, 5\right)(\bar{t}=x, \bar{x}=y)$ whereas $R\left(2 A_{2.1}, 7\right)$ is missing in [6].

(iv) $N u\left(A_{3,1} \oplus A_{1}\right) \sim\left(A_{3.1} \oplus A_{1}\right)$. No realization exists in $(1+1)$-dimension.

(v) $N u\left(A_{3,2} \oplus A_{1}, f(x)=0\right) \sim\left(A_{3.2} \oplus A_{1}\right)$. $N u\left(A_{3,2} \oplus A_{1}, f(x)=0\right) \sim R\left(A_{3.2} \oplus A_{1}, 9\right)(\bar{t}=y$, $\bar{x}=-x)$.

(vi) $N u\left(A_{3,3} \oplus A_{1}\right) \sim\left(A_{3.3} \oplus A_{1}\right)$. No realization exists in $(1+1)$-dimension. (vii) $N u\left(A_{3,4} \oplus A_{1}, f(x)=0\right) \sim\left(A_{3.4}^{a} \oplus A_{1}, a=-1\right)$.

$N u\left(A_{3,4} \oplus A_{1}, f(x)=0\right) \sim R\left(A_{3.4}^{a} \oplus A_{1}, 9, a=-1\right)$ $\left(\bar{t}=y, \bar{x}=x^{2}\right)$.

(viii) $N u\left(A_{3,5}^{a} \oplus A_{1}, 0<|a|<1, f(x)=0\right) \sim\left(A_{3.4}^{a} \oplus\right.$ $\left.A_{1},|a| \leq 1, a \neq 0, \pm 1\right)$.

$N u\left(A_{3,5}^{a} \oplus A_{1}, 0<|a|<1, f(x)=0\right) \sim R\left(A_{3.4}^{a} \oplus\right.$ $\left.A_{1}, 9\right)\left(\bar{t}=y, \bar{x}=x^{1-a}\right)$.

(ix) $N u\left(A_{3,6} \oplus A_{1}, f(x)=0\right) \sim\left(A_{3.5}^{b} \oplus A_{1}, b=0\right)$. $N u\left(A_{3,6} \oplus A_{1}, f(x)=0\right) \sim R\left(A_{3.5}^{b} \oplus A_{1}, 8, b=0\right)$ $\left(\bar{t}=y, \bar{x}=\left(x^{2}-1\right)^{1 / 2}\right)$.

(x) $N u\left(A_{3,7}^{b} \oplus A_{1}, b>0, f(x)=0\right) \sim\left(A_{3.5}^{b} \oplus A_{1}, b>0\right)$. $N u\left(A_{3,7}^{b} \oplus A_{1}, b>0, f(x)=0\right) \sim R\left(A_{3.5}^{b} \oplus A_{1}, 8\right.$, $b>0)(\bar{t}=y, \bar{x}=x)$.

(xi) $N u\left(A_{3,8} \oplus A_{1}, f(x)=1\right) \sim\left(\operatorname{Sl}(2, \mathbb{R}) \oplus A_{1}\right)\left(\bar{e}_{1}=e_{1}\right.$, $\left.\bar{e}_{2}=e_{2}, \bar{e}_{3}=-e_{3}, \bar{e}_{4}=e_{4}\right)$.

$N u\left(A_{3,8} \oplus A_{1}, b>0, f(x)=1\right) \sim R(\operatorname{Sl}(2, \mathbb{R}) \oplus$ $\left.A_{1}, 9\right)(\bar{t}=y, \bar{x}=x)$ whereas $\left.R\left(\operatorname{Sl}(2, \mathbb{R}) \oplus A_{1}, 8\right)\right)$ is missing in [6].

(xii) $N u\left(A_{3.9} \oplus A_{1}\right) \sim\left(\operatorname{So}(3) \oplus A_{1}\right)$. No realization exists in $(1+1)$-dimension.

(xiii) $N u\left(A_{4,1}, f(x)=0\right) \sim\left(A_{4.1}\right)$. $N u\left(A_{4,1}, f(x)=0\right) \sim R\left(A_{4.1}, 8\right)(\bar{t}=y, \bar{x}=x)$.

(xiv) $N u\left(A_{4,2}^{b}, b \neq 0,1\right) \sim\left(A_{4.2}^{b}, b \neq 0,1\right),\left(\bar{e}_{1}=e_{1}, \bar{e}_{2}=-e_{2}\right.$, $\left.\bar{e}_{3}=-e_{3}, \bar{e}_{4}=e_{4}\right)$.

$N u\left(A_{4,2}^{b}\right) \sim R\left(A_{4.2}^{b}, 8\right)\left(\bar{t}=e^{(b-1) x} y, \bar{x}=e^{(b-1) x}\right)$.

(xv) $N u\left(A_{4.2}^{1}\right) \sim\left(A_{4.2}^{b}, b=1\right)$. No realization exists in $(1+$ 1)-dimension.

(xvi) $N u\left(A_{4,3}, f(x)=0\right) \sim\left(A_{4.3}\right)$. $N u\left(A_{4,3}, f(x)=0\right) \sim R\left(A_{4.3}, 8\right)(\bar{t}=y, \bar{x}=x)$.

(xvii) $N u\left(A_{4,4}, f(x)=0\right) \sim\left(A_{4.4}\right)$. $N u\left(A_{4,4}, f(x)=0\right) \sim R\left(A_{4.4}, 7\right)(\bar{t}=y, \bar{x}=x)$.

(xviii) $N u\left(A_{4,5}^{a, b},-1 \leq a<b<1, a b \neq 0\right) \sim\left(A_{4,5}^{a, b},-1 \leq\right.$ $a<b<c=1, a b c \neq 0)\left(\bar{e}_{1}=e_{2}, \bar{e}_{2}=e_{3}, \bar{e}_{3}=e_{1}\right.$, $\left.\bar{e}_{4}=e_{4}\right)$.

$N u\left(A_{4,5}^{a, b},-1 \leq a<b<1, a b \neq 0\right) \sim R\left(A_{4,5}^{a, b}, 7,-1 \leq\right.$ $a<b<c=1, a b c \neq 0, b>0$ if $a=-1)\left(\bar{t}=x^{a-1} y+\right.$ $\left.x^{a} \ln (1 /|x|), \bar{x}=\ln |x|\right)$.

(xix) $\left(A_{4,5}^{a, a}, \quad-1 \leq a<1, a \neq 0\right) \sim\left(A_{4.5}^{1,1, a^{-1}},-1 \leq a<1\right.$, $a \neq 0),\left(\bar{e}_{1}=e_{3}, \bar{e}_{2}=e_{2}, \bar{e}_{3}=e_{1}, \bar{e}_{4}=e_{4}\right)$. No realization exists in $(1+1)$-dimension.

(xx) $N u\left(A_{4,5}^{a, 1},-1 \leq a<1, a \neq 0\right) \sim\left(A_{4.5}^{1,1, a},-1 \leq a<1\right.$, $a \neq 0),\left(\bar{e}_{1}=e_{1}, \bar{e}_{2}=e_{3}, \bar{e}_{3}=e_{2}, \bar{e}_{4}=e_{4}\right)$. No realization exists in $(1+1)$-dimension.

(xxi) $N u\left(A_{4,5}^{1,1}\right) \sim\left(A_{4,5}^{a, b}, a=b=c=1\right)\left(\bar{e}_{1}=e_{2}, \bar{e}_{2}=e_{3}\right.$, $\left.\bar{e}_{3}=e_{1}, \quad \bar{e}_{4}=e_{4}\right)$.

$N u\left(A_{4,5}^{1,1}\right) \sim R\left(A_{4,5}^{a, b}, 10, a=b=c=1\right)(\bar{t}=y$, $\bar{x}=x)$.

(xxii) $N u\left(A_{4,6}^{a, b}, a \neq 0, b \geq 0\right) \sim\left(A_{4.6}^{a, b}, a>0\right),\left(\bar{e}_{1}=e_{1}\right.$, $\left.\bar{e}_{2}=e_{3}, \quad \bar{e}_{3}=-e_{2}, \quad \bar{e}_{4}=e_{4}\right)$.

$N u\left(A_{4,6}^{a, b}, a \neq 0, b \geq 0\right) \sim R\left(A_{4.6}^{a, b}, 6, a>0\right)(\bar{t}=$ $\left.y\left(1+x^{2}\right)^{-1 / 2} e^{(a-b) \tan ^{-1} x}, \bar{x}=\tan ^{-1} x\right)$. 
TABLE 1

\begin{tabular}{|c|c|c|}
\hline Lie algebra & $N$ & Realizations (generators) \\
\hline $4 A_{1}$ & 11 & $\partial_{t}, \quad x \partial_{t}, \quad k(x) \partial_{t}, h(x) \partial_{t}$ \\
\hline $2 A_{2.1}$ & $\begin{array}{l}5 \\
* 7\end{array}$ & $\begin{array}{c}\partial_{t}, t \partial_{t}, \partial_{x}, x \partial_{x} \\
\partial_{t}, t \partial_{t}+x \partial_{x}, x \partial_{t}, \quad-x \partial_{x}\end{array}$ \\
\hline$A_{3.2} \oplus A_{1}$ & 9 & $\partial_{t}, x \partial_{t}, t \partial_{t}-\partial_{x}, e^{-x} \partial_{t}$ \\
\hline$A_{3.4}^{a} \oplus A_{1},|a| \leq 1, a \neq 0,1$ & 9 & $\partial_{t}, \quad x \partial_{t}, \quad t \partial_{t}+(1-a) x \partial_{x}, \quad|x|^{1 /(1-a)} \partial_{t}$ \\
\hline$A_{3.5}^{b} \oplus A_{1}, b \geq 0$ & 8 & $\partial_{t}, \quad x \partial_{t}, \quad(b-x) t \partial_{t}-\left(1+x^{2}\right) \partial_{x}, \quad \sqrt{1+x^{2}} e^{-b \arctan x} \partial_{t}$ \\
\hline $\mathrm{Sl}(2, \mathbb{R}) \oplus A_{1}$ & $\begin{array}{l}{ }^{*} 8 \\
9\end{array}$ & $\begin{array}{c}\partial_{t}, t \partial_{t}+x \partial_{x}, t^{2} \partial_{t}+2 t x \partial_{x}, x \partial_{x} \\
\partial_{t}, t \partial_{t}, t^{2} \partial_{t}, \partial_{x}\end{array}$ \\
\hline$A_{4.1}$ & 8 & $\partial_{t}, x \partial_{t},(1 / 2) x^{2} \partial_{t},-\partial_{x}$ \\
\hline$A_{4.2}^{b}, \quad b \neq 0$ & 8 & $\partial_{t}, \quad x \partial_{t}, \quad(1 /(1-b)) x \ln |x| \partial_{t}, \quad b t \partial_{t}+(b-1) x \partial_{x}, b \neq 1$ \\
\hline$A_{4.3}$ & 8 & $\partial_{t}, \quad x \partial_{t}, \quad-x \ln |x| \partial_{t}, \quad t \partial_{t}+x \partial_{x}$ \\
\hline$A_{4.4}$ & 7 & $\partial_{t}, x \partial_{t},(1 / 2) x^{2} \partial_{t}, t \partial_{t}-\partial_{x}$ \\
\hline$A_{4.5}^{a, b, c},-1 \leq a<b<c=1, a b c \neq 0$ & $\begin{array}{c}7 \\
10\end{array}$ & $\begin{array}{c}\partial_{t}, \quad e^{(a-b) x} \partial_{t}, \quad e^{(a-1) x} \partial_{t}, \quad a t \partial_{t}+\partial_{x}, b>0 \text { if } a=-1 \\
\partial_{t}, \quad x \partial_{t}, \quad \phi(x) \partial_{t}, \quad t \partial_{t}, \phi^{\prime \prime}(x) \neq 0, \quad a=b=c=1\end{array}$ \\
\hline$A_{4.6}^{a, b}, a>0$ & 6 & $\partial_{t}, \quad e^{(a-b) x} \cos x \partial_{t}, \quad-e^{(a-b) x} \sin x \partial_{t}, \quad a t \partial_{t}+\partial_{x}$ \\
\hline$A_{4.7}$ & 5 & $\partial_{t}, \quad x \partial_{t}, \quad-\partial_{x}, \quad\left(2 t-(1 / 2) x^{2}\right) \partial_{t}+x \partial_{x}$ \\
\hline$A_{4.8}^{b},|b| \leq 1$ & $\begin{array}{l}5 \\
{ }^{*} 7\end{array}$ & $\begin{array}{c}\partial_{t}, \partial_{x}, \quad x \partial_{t}, \quad(1+b) t \partial_{t}+x \partial_{x} \\
\partial_{t}, \quad x \partial_{t}, \quad-\partial_{x}, \quad(1+b) t \partial_{t}+b x \partial_{x}, \quad b \neq \pm 1\end{array}$ \\
\hline$A_{4.10}$ & $\begin{array}{c}{ }^{*} 6 \\
7\end{array}$ & $\begin{array}{c}\partial_{t}, \partial_{x}, \quad t \partial_{t}+x \partial_{x}, \quad x \partial_{t}-t \partial_{x} \\
\partial_{t}, \quad x \partial_{t}, \quad t \partial_{t}, \quad-t x \partial_{t}-\left(1+x^{2}\right) \partial_{x}\end{array}$ \\
\hline
\end{tabular}

(xxiii) $N u\left(A_{4,7}\right) \sim\left(A_{4.7}\right)$. $N u\left(A_{4,7}\right) \sim R\left(A_{4.7}, 5\right)\left(\bar{t}=y+\left(x^{2} / 4\right)(1-2 \log |x|)\right.$, $\bar{x}=x)$.

(xxiv) $N u\left(A_{4,8}\right) \sim\left(A_{4.8}^{b},|b| \leq 1, b=-1\right)$. $N u\left(A_{4,8}\right) \sim R\left(A_{4.8}^{b},|b| \leq 1,5, b=-1\right)(\bar{t}=y$, $\bar{x}=x)$.

$(\mathrm{xxv}) \operatorname{Nu}\left(A_{4,9}^{b}, 0<|b|<1\right) \sim\left(A_{4.8}^{b},|b| \leq 1, \quad b \neq \pm 1,0\right)$. $N u\left(A_{4,9}^{b}\right) \sim R\left(A_{4.8}^{b}, 5\right)(\bar{t}=y, \bar{x}=x)$ whereas $R$ $\left(A_{4.8}^{b}, 7, b \neq \pm 1,0\right)$ is missing in [6].

(xxvi) $N u\left(A_{4,9}^{1}\right) \sim\left(A_{4.8}^{b}, b=1\right)$. $N u\left(A_{4,9}^{1}\right) \sim R\left(A_{4.8}^{1}, 5\right)(\bar{t}=y, \bar{x}=x)$.

(xxvii) $N u\left(A_{4,9}^{0}\right) \sim\left(A_{4.8}^{b}, b=0\right)$. $N u\left(A_{4,9}^{0}\right) \sim R\left(A_{4.8}^{0}, 5\right)(\bar{t}=y, \bar{x}=x)$ whereas $R$ $\left(A_{4.8}^{b}, 7, b=0\right)$ is missing in [6].

(xxviii) $N u\left(A_{4.10}\right) \sim\left(A_{4.9}^{a}, a=0\right)$. No realization exists in $(1+1)$-dimension.

(xxix) $N u\left(A_{4.11}^{a}, a>0\right) \sim\left(A_{4.9}^{a}, a>0\right)$. No realization exists in $(1+1)$-dimension. $(\mathrm{xxx}) N u\left(A_{4,12}\right) \sim\left(A_{4.10}\right)$. $N u\left(A_{4,12}\right) \sim R\left(A_{4.10}, 7\right)(\bar{t}=y, \bar{x}=x)$ whereas $R\left(A_{4.10}, 6\right)$ is missing in [6].

Remarks for Table 1

(i) $4 A_{1}: 1, x, h(x)$ and $k(x)$ form a linearly independent set.

Remarks for Tables 1 and 2. In both tables we have

(i) $h, k$, and $\phi$ are arbitrary functions with specified conditions mentioned in the corresponding realizations.

(ii) $a, b$, and $c$ are parameters and arbitrary constants, whose range and values are mentioned in each of the realizations.

(iii) $*$ : these are the cases of realizations which are missing in $[6]$.

Remarks for Table 2

(i) *: these are the canonical forms of fourth-order ODEs which are missing in [6]. Only these are given here with their corresponding algebras and realizations. 
TABLE 2

\begin{tabular}{|c|c|c|}
\hline Lie algebra & $N$ & Realizations and equations \\
\hline $2 A_{2.1}$ & $* 7$ & $\begin{array}{c}\partial_{t}, t \partial_{t}+x \partial_{x}, x \partial_{t},-x \partial_{x} \\
\cdots=\frac{10 \ddot{x} \ddot{x}}{\dot{x}}-\frac{15 \ddot{x}^{3}}{\dot{x}^{2}}+\frac{\dot{x}^{2} \ddot{x}}{x^{2}} f\left(\frac{x \ddot{x}}{\dot{x} \ddot{x}}-\frac{3 x \ddot{x}}{\dot{x}^{2}}\right)\end{array}$ \\
\hline $\operatorname{Sl}(2, \mathbb{R}) \oplus A_{1}$ & ${ }^{*} 8$ & $\begin{array}{c}\partial_{t}, t \partial_{t}+x \partial_{x}, t^{2} \partial_{t}+2 t x \partial_{x}, x \partial_{x} \\
\cdots=-\frac{2 \dot{x} \ddot{x}}{x}+\frac{1}{x^{3}}\left(\frac{\dot{x}^{2}}{2}-x \ddot{x}\right)^{2} f\left(\frac{x^{2} \ddot{x}}{\left(\left(\dot{x}^{2} / 2\right)-x \ddot{x}\right)^{3 / 2}}\right)\end{array}$ \\
\hline$A_{4.8}^{b},|b| \leq 1$ & ${ }^{*} 7$ & $\begin{array}{c}\partial_{t}, x \partial_{t},-\partial_{x},(1+b) t \partial_{t}+b x \partial_{x}, b \neq \pm 1 \\
\dddot{x}=\frac{10 \ddot{x} \dddot{x}}{\dot{x}}-\frac{15 \ddot{x}^{3}}{\dot{x}^{2}}+\ddot{x}^{(1-3 b) /(1-b)} \dot{x}^{(4-2 b) /(1-b)} f\left(\left(\frac{\ddot{x}}{\dot{x}^{4}}-\frac{3 \ddot{x}^{2}}{\dot{x}^{5}}\right)\left(\frac{\ddot{x}}{\dot{x}^{3}}\right)^{(1-2 b) /(b-1)}\right)\end{array}$ \\
\hline$A_{4.10}$ & *6 6 & $\begin{array}{c}\partial_{t}, \partial_{x}, t \partial_{t}+x \partial_{x}, x \partial_{t}-t \partial_{x} \\
\dddot{x}=\frac{10 \dot{x} \ddot{x} \ddot{x}}{\left(1+\dot{x}^{2}\right)}-\frac{15 \dot{x}^{2} \ddot{x}^{3}}{\left(1+\dot{x}^{2}\right)^{2}}+\frac{\ddot{x}^{3}}{\left(1+\dot{x}^{2}\right)^{2}} f\left(\frac{\left(1+\dot{x}^{2}\right) \dddot{x}}{\ddot{x}^{2}}-3 \dot{x}\right)\end{array}$ \\
\hline
\end{tabular}

\section{Concluding Remarks}

In this contribution we have supplemented the work [6] for the canonical forms of scalar fourth-order ODEs and have obtained four new forms as listed in Table 2. The integrability of these equations has the same route as the others which are discussed at length in [6].

\section{Acknowledgments}

A. Fatima gratefully acknowledges the financial support and scholarship from School of Computational and Applied Mathematics, University of the Witwatersrand.

\section{References}

[1] S. Lie, "Classification und Integration von gewöhnlichen Differentialgleichungen zwischen $x, y$, die eine Gruppe von Transformationen gestatten," Mathematische Annalen, vol. 8, no. 9, p. $187,1888$.

[2] P. J. Olver, Applications of Lie Groups to Differential Equations, vol. 107, Springer, New York, NY, USA, 2nd edition, 1993.

[3] A. H. Kara, F. M. Mahomed, and P. G. L. Leach, "Noether equivalence problem for particle Lagrangians," Journal of Mathematical Analysis and Applications, vol. 188, no. 3, pp. 867-884, 1994.

[4] F. M. Mahomed and P. G. L. Leach, "Normal forms for thirdorder equations," in Proceedings of the Workshop on Finite Dimensional Integrable Nonlinear Dynamical Systems, P. G. L. Leach and W. H. Steeb, Eds., p. 178, World Scientic, Johannesburg, South Africa, 1988.

[5] N. H. Ibragimov and M. C. Nucci, "Integration of third order ordinary differential equations by lies method: equations admitting three-dimensional lie algebras," Lie Groups and Their Applications, vol. 1, p. 4964, 1994.

[6] T. Cerquetelli, N. Ciccoli, and M. C. Nucci, "Four dimensional Lie symmetry algebras and fourth order ordinary differential equations," Journal of Nonlinear Mathematical Physics, vol. 9, supplement 2, pp. 24-35, 2002.

[7] J. Patera and P. Winternitz, "Subalgebras of real three- and fourdimensional Lie algebras," Journal of Mathematical Physics, vol. 18, no. 7, pp. 1449-1455, 1977.
[8] F. M. Mahomed and P. G. L. Leach, "Lie algebras associated with scalar second-order ordinary differential equations," Journal of Mathematical Physics, vol. 30, no. 12, pp. 2770-2777, 1989.

[9] R. O. Popovych, V. M. Boyko, M. O. Nesterenko, and M. W. Lutfullin, "Realizations of real low-dimensional Lie algebras," Journal of Physics A, vol. 36, no. 26, pp. 7337-7360, 2003.

[10] G. Bluman and S. Kumei, "On invariance properties of the wave equation," Journal of Mathematical Physics, vol. 28, no. 2, pp. 307-318, 1987.

[11] S. P. Timoshenko, Theory of Elastic Stability, McGraw-Hill, New York, NY, USA, 2nd edition, 1961.

[12] A. H. Bokhari, F. M. Mahomed, and F. D. Zaman, "Symmetries and integrability of a fourth-order Euler-Bernoulli beam equation," Journal of Mathematical Physics, vol. 51, no. 5, Article ID 053517, 2010.

[13] F. I. Leite, P. D. Lea, and T. Mariano, "Symmetry and integrability of a fourth-order Emden-Fowler equation," Technical Report CMCC-UFABC1-13, 2012. 


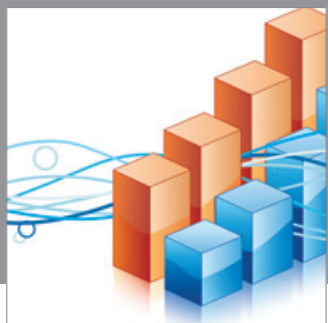

Advances in

Operations Research

mansans

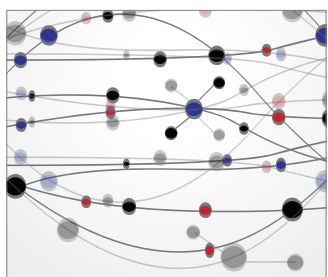

The Scientific World Journal
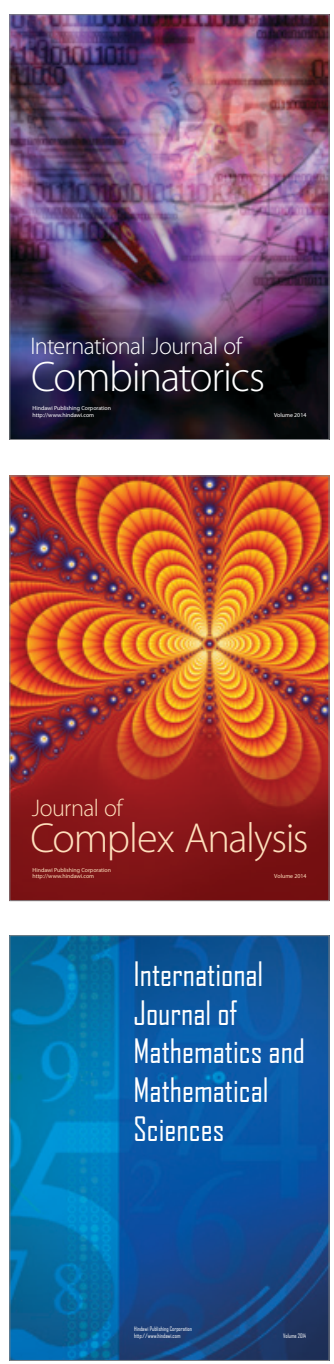
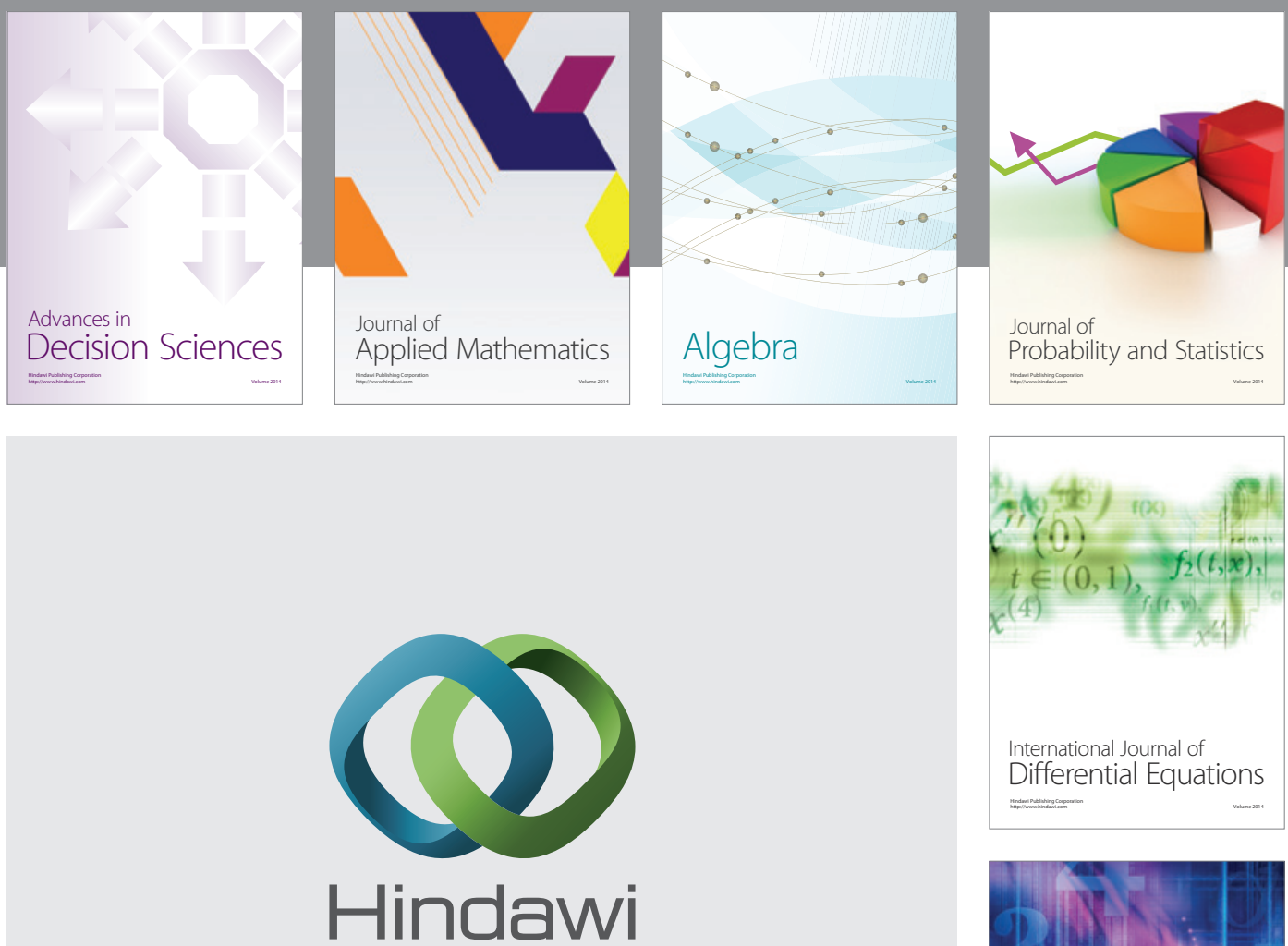

Submit your manuscripts at http://www.hindawi.com
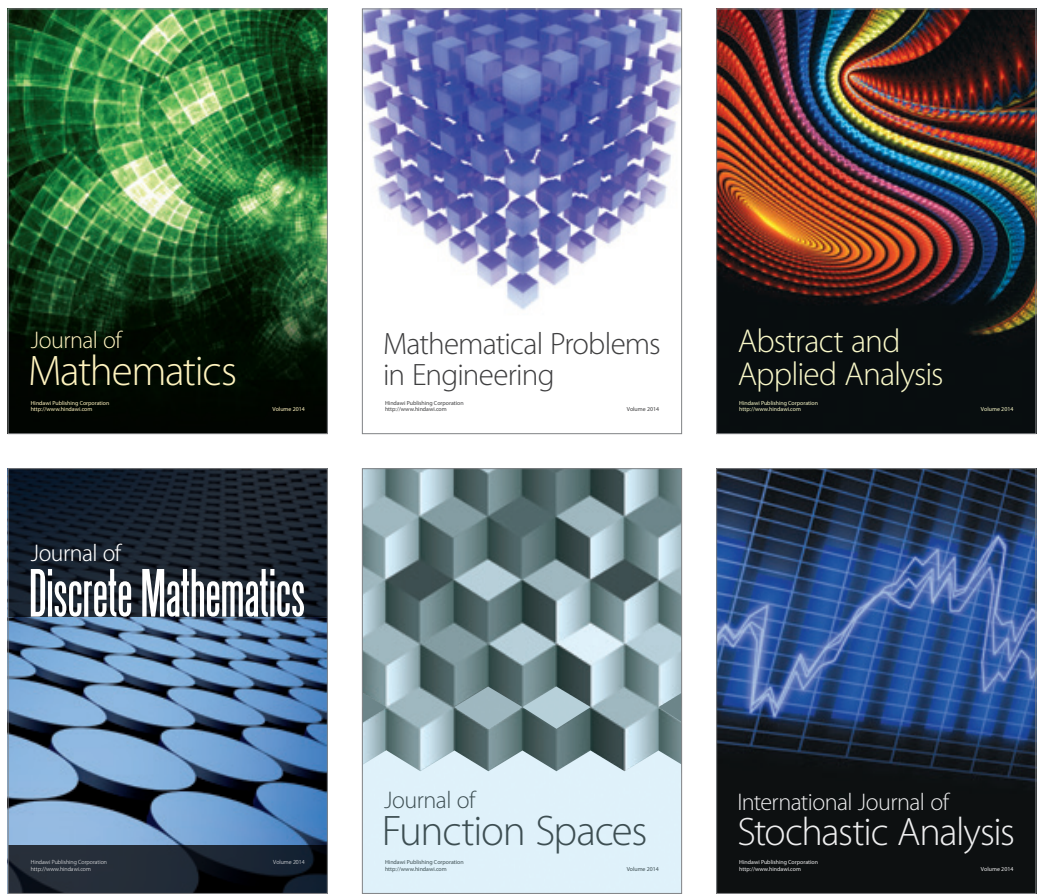

Journal of

Function Spaces

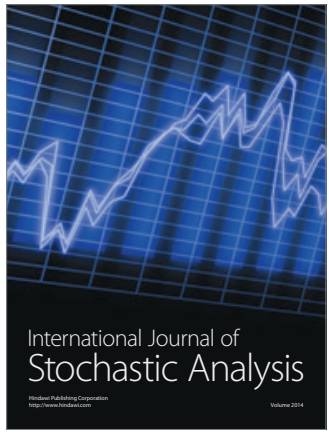

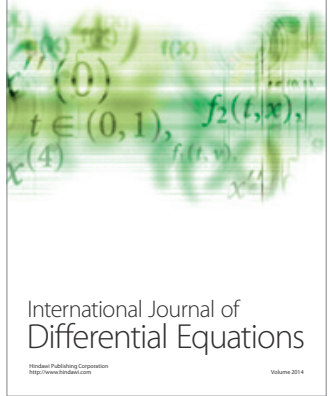
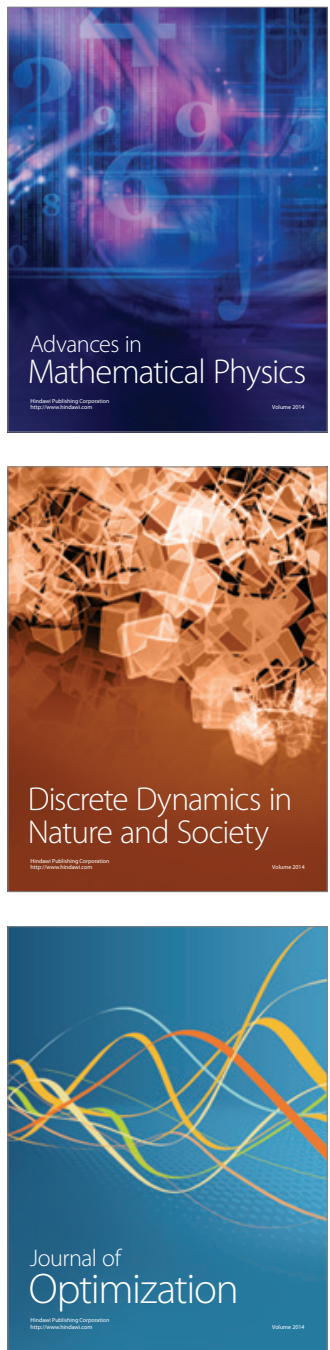